\title{
Análisis del manejo alimenticio con forraje hidropónico en bovinos de ceba en la vereda Jiramena, San Carlos de Guaroa, Colombia
}

\section{Analysis of dietary with hydroponic forage in_fattening cattle in the Jiramena, village of San Carlos de Guaroa, Colombia}

\author{
Frías Bello Carlos Francisco ${ }^{1}$, Lozada Hernando ${ }^{2}$ y Torres Joyne Raquel ${ }^{3}$ \\ ${ }^{1}$ Licenciado en Producción Agropecuaria. Universidad de los Llanos \\ ${ }^{2}$ Medico Veterinario Zootecnista, Docente Universidad de los Llanos \\ ${ }^{3}$ Antropóloga, Docente Universidad de los Llanos \\ hdolozada@yahoo.com.ar
}

Recibido 05 de Marzo 2013, Aceptado 02 de Abril de 2013

\section{RESUMEN}

El presente trabajo de investigación evalúo la producción de bovinos de ceba de esta región ya que no está permitiendo generar una rentabilidad que consiga mejorar las condiciones de vida de los pobladores; debido a que no existe una adecuada alimentación y manejo de los animales para lograr mantener una cantidad apropiada de ganado que genere las utilidades deseadas. Al analizar la información se encontró que los productores de bovinos de ceba llevan más de cinco años laborando en estas tierras y que están desarrollando esta actividad de la ganadería como reglón importante para su económica, pero que por ser de manera extensiva no está proporcionando los ingresos necesarios para suplir sus necesidades. Esto se pudo determinar porque la actividad de la ganadería no posee ninguna tecnificación, lo que supone un manejo inadecuado del desarrollo de la producción y por ende de la alimentación que se le da a los bovinos. Los resultados también arrojaron que los productores de bovinos de ceba no complementan la alimentación de los animales con otro forraje, por lo que solo emplean las pasturas que nacen de los campos propios y por ello es que la ganadería se hace extensiva; que sumado a la falta de conocimiento sobre complementación alimenticia y el poco apoyo del Estado, se forma una situación adversa que repercute en el desarrollo de la región. Por otro lado, se definió que tampoco existen los equipos ni la infraestructura adecuada para el manejo de los 
bovinos, por lo tanto, en muchos casos hay bajas en el número de semovientes, lo cual disminuye los ingresos empeorando la situación de las personas. Al realizar la comparación bromatológica de las gramíneas de pastoreo tradicional y producción de forraje verde hidropónico (FVH) como alternativa para mejorar el proceso de alimentación, se encontró que el porcentaje de proteína de una muestras de FVH a los quince días de rebrote es de $18.22 \%$, lo cual contribuye a mejorar el peso del animal en 677 gramos, un porcentaje importante para lograr mejorar el peso del animal en menor tiempo y también para tener más ganado en pequeños terrenos como los de estos productores, lo cual llevará a incrementar sus ingresos. Desarrollando la creación del montaje para la producción de FVH se pudo llegar a concluir con la evaluación financiera y con sus parámetros para ello como V.P.N. T.I.R, RB/C, y el análisis de sensibilidad considerando una tasa de oportunidad del $12 \%$, que es rentable invertir en un proyecto de esta índole porque está dejando una tasa de retorno de $32 \%$, lo que significa que el productor debe mejorar sus condiciones de producción para lograr mayores ingresos. Por lo tanto, el FVH posibilita que el ganadero cuente con un forraje verde en la cantidad deseada según la necesidad, siendo de alta calidad y aun valor sustancialmente más económico que el forraje convencional; sustituyendo así los grandes espacios de terreno que son imprescindibles para obtener forraje, creando granjas competitivas de reducidas dimensiones y altas producciones en las zonas donde el suelo y el clima son adversos.

Palabras clave: Forraje verde hidropónico, bovinos de ceba, alimentación, suplemento nutricional.

\section{ABSTRACT}

The present research evaluated the production of steers of this region as it is not allowing to generate a return that succeed in improving the living conditions of the residents, because there is no adequate feeding and handling of animals in order to maintain an appropriate amount won to generate the desired utilities. By analyzing information found that steers producers spent more than five years working on this land and who are developing this farming activity as important for 
economic screed, but that being extensively is not providing revenue necessary to meet their needs. This could be determined because the activity does not have any livestock modernization, representing the mismanagement of development of production and hence food that is given to cattle. The results also showed that cattle producers not fattening food supplement with another animal fodder, so only use pasture fields arising from own and that is why livestock is extended, which added to the lack of knowledge about food supplementation and little support from the state, formed an adverse situation that affects the development of the region. On the other hand, there are no defined teams and adequate infrastructure for handling cattle, so in many cases are low in the number of livestock, decreasing revenues worsening the situation of people. When comparing bromatological traditional grazing grasses and hydroponic forage production $(\mathrm{FVH})$ as an alternative to improve the feeding process, it was found that the protein content of the fifteenth day is $18.22 \%$, which contributes to improve the animal's weight at 677 grams, a significant percentage towards improved animal weight in less time and also to have more livestock on small plots as these producers, which will lead to increase revenue. Developing assembly building for the production of $\mathrm{FVH}$ conclusion could be reached with the financial evaluation and its parameters to it as V.P.N., T.I.R, RB/C, and the sensitivity analysis considering a chance rate of $12 \%$, which is profitable to invest in a project of this nature because it is making a return rate of $32 \%$, which means that the producer must improve their production conditions to achieve higher revenues. Therefore, the FVH enables the farmer to have a forage in the desired quantity as needed, with high quality and value still substantially cheaper than conventional feed, thus replacing large areas of land that are essential for forage, creating competitive farms of small size and high yields in areas where the soil and climate are adverse.

Keywords: Hydroponic green fodder, cattle fattening, feeding, nutritional supplement. 


\section{INTRODUCCIÓN}

El objetivo general del presente trabajo fue analizar el manejo de la alimentación de los bovinos de ceba de los productores de la Vereda Jiramena del Municipio de San Carlos de Guaroa en el Departamento del Meta, para conocer su incidencia en la producción a fin de generar una proyección de la producción de forraje verde hidropónico $(\mathrm{FVH})$ como alternativa complementaria de alimentación. El trabajo de investigación se desarrolló en razón a que, en esta región, la producción de bovinos de ceba está dejando poca rentabilidad porque no existe una adecuada alimentación y manejo de los animales para lograr mantener una cantidad apropiada de carga animal por hectárea en cada una de las fincas, que genere unas utilidades que suplan las necesidades del ganadero. Por ello, prefiere salir del campo para desplazarse a la ciudad aumentando el problema social, que repercute directamente en el desarrollo de la región.

Por lo tanto la situación problema, radica en la ausencia de un manejo adecuado de alimentación para los bovinos de ceba, que proviene de la falta de capacitación e información de los productores y su interés por desarrollar alternativas viables para el mejoramiento o producción de pasturas a partir de un sistema sostenible que no sea costoso y que le permita al ganadero mantener en condiciones adecuadas una cantidad de bovinos apropiada para que su área de trabajo sea eficiente y rentable y le otorgue los ingresos necesarios para cubrir sus expectativas de vida.

Es claro apreciar, que además de la conformación del suelo que se posee en los Llanos Orientales, existen otras causas que están generando la desaparición de las plantas forrajeras, tales como la utilización de especies no adaptadas, presencia de plagas, y la falta de fertilización; con el tiempo aparecen en las praderas aéreas descubiertas que son más susceptibles a la compactación por el pisoteo del animal, afectando las características del suelo, impidiendo el normal desarrollo radicular por la reducción de los espacios porosos, deficiencia de oxígeno, disminución de la infiltración y aumento de la escorrentía, factores que favorecen la erosión de los suelos. 
A estos hechos se le suma el que los productores en muchos casos crean una forma de ganadería extensiva que conlleva a desmejorar la calidad de bovinos en peso y por supuesto en carne la cual no presentara las mismas condiciones, de un animal que tuvo una buena alimentación. Esto representa para el productor bajos ingresos, daños en las pasturas, y la formación de los factores antes mencionados que repercuten directamente en el ambiente y ecosistema en donde se desarrollan estas actividades, pues muchas de las praderas con grandes paisajes se han convertido en tierras áridas y sin las características propias de la región que se desea.

Es importante entender que, en la región al igual que en el resto del país se requieren de fuerzas que generen un alto crecimiento económico y un bienestar general para sus habitantes, con el único objetivo de contribuir con el progreso y desarrollo económico; se considera, que uno de los factores claves del surgimiento, es el proponer iniciativas enfocadas al mejoramiento de situaciones adversas que afectan a la población de una u otra forma. $\mathrm{Y}$, es aquí en donde se empieza a establecer la importancia de los proyectos que nacen como parte de la búsqueda de una solución inteligente a una situación, problema o a una necesidad humana y que a su vez pueden contribuir a resolver las dificultades o cambiar las condiciones existentes. Por eso el objetivo del trabajo fue analizar el manejo de la alimentación de los bovinos de ceba de los productores de la Vereda Jiramena del Municipio de San Carlos de Guaroa en el Departamento del Meta, para conocer su incidencia en la producción a fin de generar una proyección de la producción de forraje verde hidropónico $(\mathrm{FVH})$ como alternativa complementaria.

La región de la Orinoquía colombiana presenta grandes opciones en el campo de la agricultura y la ganadería y de hecho se evidencia gran aceptación o acogida en las diferentes ideas novedosas que se enfocan a mejorar las situaciones adversas que se dan en muchos casos por la falta de información, capacitación y de apoyo de las diferentes instituciones del Estado, el cual tiene la misión de suplir las necesidades y expectativas de una población que busca mejorar su nivel de vida en todo momento. Este trabajo se enfoca en el tema de la ganadería de ceba 
como actividad esencial para los productores de la Vereda Jiramena del Municipio de San Carlos de Guaroa del Departamento del Meta, en relación con la importancia de mejorar las condiciones de la alimentación de los bovinos de ceba, a través de una alternativa complementaria, como lo es la producción de FVH que busca suplir la necesidad de pastos producidos en la región debido a que los suelos no poseen las condiciones más favorables para su desarrollo y su manejo por parte de los productores no es él más óptimo. Esto ha generado una serie de consecuencias para el productor en relación con la cantidad de bovinos que se pueden cebar en las diferentes fincas, lo cual lleva a que las condiciones de vida de la población no sean las mejores y no se presenten opciones de expectativas de vida hacia el futuro en esta área.

También, se encontró que la capacitación sobre el tema es mínima y que existe muy poco apoyo del estado, lo cual no permite mejorar la calidad de vida de la población, por lo tanto, siguen con unas condiciones no favorables para suplir sus necesidades básicas y expectativas de vida. Por otro lado, se definió también que no existen los equipos ni la infraestructura adecuada para el manejo de los bovinos y por lo tanto en muchos casos hay bajas en el número de semovientes, lo cual disminuye los ingresos empeorando la situación de las personas.

En el caso del $\mathrm{FVH}$, la población posee pocos conocimientos y no conocen las ventajas de este cultivo como alimento complementario importante para el desarrollo de los animales, por lo cual la capacitación es fundamental como alternativa de solución a esta situación.

\section{METODOLOGÍA}

Por la magnitud del problema se inscribe dentro del tipo investigación descriptiva, ya que su objetivo es reseñar los fenómenos, estado, características y actividades que en forma natural desarrollan los productores de bovinos de ceba en la Vereda Jiramena del Municipio de San Carlos de Guaroa en el Departamento del Meta.

Se llevo a cabo los siguientes tipos de análisis: se caracterizó globalmente el objeto de estudio por medio de tablas y gráficas estadísticas, que permitieron 
mostrar aspectos del manejo de los bovinos de ceba por parte de los productores en esta vereda y a la vez se realizó un análisis descriptivo, que se hizo a partir de la observación directa lo cual permitió establecer las características del tema abordado y llevarlos a un ordenamiento de estos fenómenos. Es decir, aquí, se registraron las observaciones del fenómeno en estudio como un conjunto de datos y conceptos los cuales fueron descritos de acuerdo a criterios establecidos del tema estudiado mediante un proceso de conocimiento que se inició con la observación. Por lo tanto, es importante decir que los informes y las conclusiones que fueron extraídas de las encuestas, tienen un alto grado de certeza debido a la representatividad de la forma técnica y práctica con que se aplicó. La encuesta fue diseñada en atención a los objetivos planteados, y sus preguntas se elaboraron para una fácil comprensión y análisis de los datos que se recogieron. Por medio de observación directa, se determinó, si estos procesos cuentan con la infraestructura adecuada para el manejo de los bovinos como son las instalaciones y su espacio utilizado; así mismo se pudo apreciar la actitud de los productores y su interés por mejorar las condiciones existentes en su área de trabajo, así como del aprovechamiento continuo del mismo para la generación de ingresos importantes para el desarrollo de vida.

La población objeto del proceso investigativo, fueron todos productores de bovinos de ceba en la Vereda Jiramena del Municipio de San Carlos de Guaroa en el Departamento del Meta, a los cuales se les aplicó las encuestas sobre el tema. Según los datos obtenidos son aproximadamente 60 productores a los cuales se les tomó una muestra. Para la realización de la muestra se seleccionó a los productores de bovinos de ceba. En dicha muestra se efectuó la medición y la observación de los factores de relevantes del tema. Para determinar el tamaño de la muestra se calculó a través de la fórmula con criterios estadísticos aplicando el método de muestreo aleatorio simple, es decir, al azar. 


$$
\mathrm{n}=\frac{\mathrm{N} \cdot \mathrm{C} 2 \cdot \mathrm{P} \cdot \mathrm{Q}}{\mathrm{e} 2 \cdot(\mathrm{N}-1)+\mathrm{C} 2 \cdot \mathrm{p} \cdot \mathrm{Q}}
$$

Donde: $\mathrm{n}=$ Tamaño de la muestra; $\mathrm{N}=$ Población (60 productores); $\mathrm{C} 2=1,96$ Constante que no debe ser menor del 95\%; e2 = 0,05 Error máximo admisible; $p=$ 0,50 Probabilidad a favor; $q=0,50$ Probabilidad en contra

Al desarrollar la fórmula se obtuvo que:

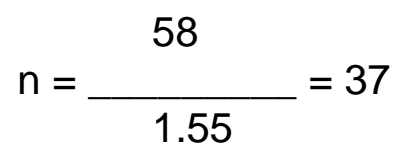

Luego, las encuestas que se realizaron fueron 37 a los productores de bovinos de ceba en la Vereda Jiramena del Municipio de San Carlos de Guaroa en el Departamento del Meta. Se llevo a cabo una observación directa, y a la vez en las visitas se indicó la importancia a los productores de recoger la información para este estudio, de los diferentes aspectos del manejo de alimentación de los bovinos de ceba y los recursos físicos con que se cuentan, el proceso de organización de los productores y otros aspectos más, que generaron una gran cantidad de variables que fueron apreciables en la realización del diagnóstico.

Los productores de bovinos están ubicados en diferentes fincas que se encuentran en este sector y corresponden a aquellas personas que están encargadas del manejo de los animales y por supuesto de su desarrollo. El presente diagnóstico se llevó a cabo con base en la recopilación y análisis de datos suministrados por los productores y la posible información documentada que poseen. Este diagnóstico, se apoyó en documentos, se obtuvo información fundamental de conceptos y datos a través de revistas, libros, folletos, periódicos documentos e Internet y normas relacionadas con el tema de investigación

La capacitación propuesta hacia los productores de bovinos de ceba en la Vereda Jiramena del Municipio de San Carlos de Guaroa en el Departamento del Meta, se realizó debido a la necesidad de generar la importancia de mejorar las condiciones del manejo alimenticio de los animales que ellos cuidan; porque de ello depende 
su economía y su mejoramiento del nivel de vida. Se emplearon métodos que buscan que los participantes aseguren la participación activa con el fin de que se genere un cambio en sus actividades, a partir de la obtención de un conocimiento sobre alimentación complementaria como lo es el $\mathrm{FVH}$, que es fácil de cultivar y con alto contenido proteínico que permite ganar en peso y calidad en los bovinos.

\section{RESULTADOS Y DISCUSIÓN}

Se evaluó el manejo de la alimentación de los bovinos de ceba que emplean los productores de la Vereda Jiramena del Municipio de San Carlos de Guaroa en el Departamento del Meta, y su incidencia en el aspecto económico de los mismos, el número de animales producido, y su efecto en el medio ambiente, lo cual fue importante en la proyección de la propuesta de producción de FVH y de la capacitación al respecto para los productores como una alternativa viable para mejorar estos aspectos relacionados.

Se efectúo la recolección de información con la cual se llevó a cabo el análisis del manejo de la alimentación de los bovinos, señalando que el reglón económico para su sustento es la ganadería de ceba, la cual desarrollan sin ninguna tecnificación, indicando que existe un manejo inadecuado del desarrollo de las tareas de estos procesos, que sumado a una falta de complementación alimenticia de los bovinos, no permiten ganar un buen peso y además no permiten poseer una cantidad importante de ganado haciendo que la producción sea extensiva y no lucrativa ya que los terrenos son de dimensiones pequeñas.

También, se encontró que la capacitación sobre el tema es mínima y que existe muy poco apoyo del Estado, lo cual no permite mejorar la calidad de vida de la población, por lo tanto, siguen con unas condiciones no favorables para suplir sus necesidades básicas y expectativas de vida. Por otro lado, se definió también que no existen los equipos ni la infraestructura adecuada para el manejo de los bovinos y por lo tanto en muchos casos hay bajas en el número de semovientes, lo cual disminuye los ingresos empeorando la situación de las personas. 
En el caso del FVH, la población posee pocos conocimientos y no conocen las ventajas de este cultivo como alimento complementario importante para el desarrollo de los animales, por lo cual la capacitación es fundamental como alternativa de solución a esta situación. Ahora, al realizar la comparación bromatológica del material utilizado para la ceba de bovinos, gramíneas de pastoreo tradicional y producción de FVH como alternativa para mejorar el proceso de alimentación, se encontró que porcentaje de proteína de una muestra de $\mathrm{FVH} \mathrm{a}$ los quince días es de $18.22 \%$, lo cual contribuye a mejorar el peso del animal en 677 gramos, 5.5 veces más que lo que produce la pastura normal, importante para solucionar la situación adversa.

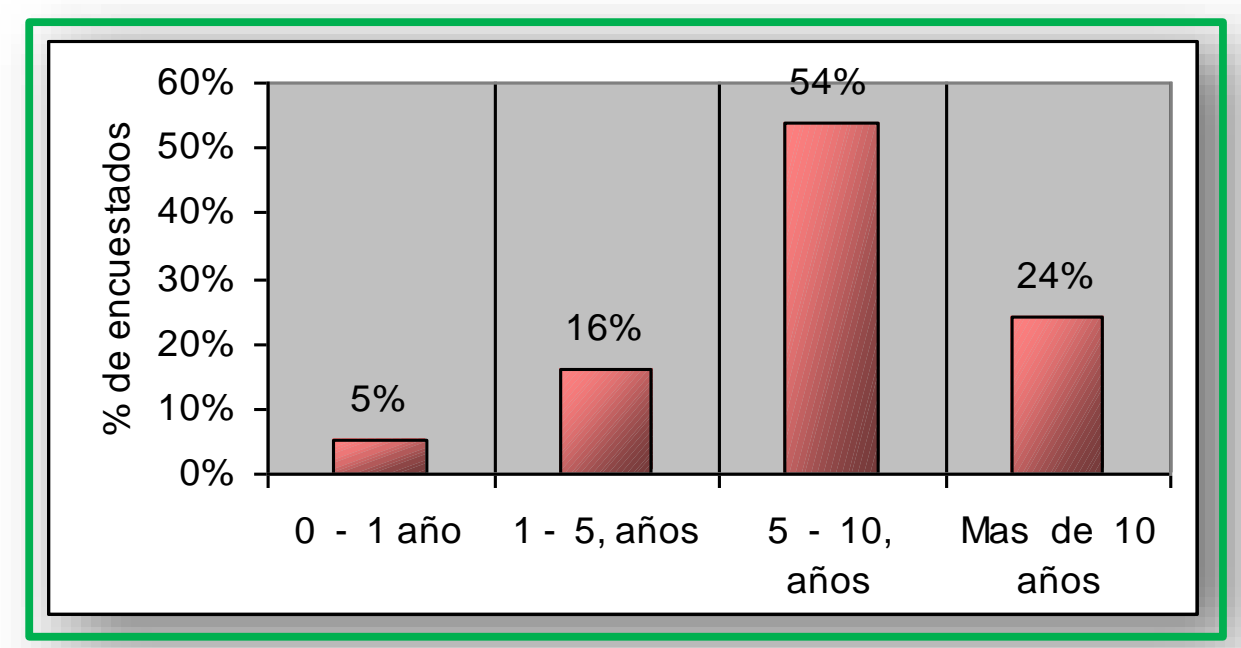

Gráfica 1. ¿Qué tiempo lleva cebando bovinos en la Vereda?

Se ve claramente que los productores de bovinos de ceba en un $54 \%$ llevan entre cinco a diez años laborando en estas tierras y desarrollando esta actividad importante para su económica. Así mismo se encontró que el $24 \%$ de los productores llevan más de 10 años cebando bovinos como medio para proporcionar los ingresos necesarios para subsistir.

La Gráfica 2 muestra que el $86 \%$ de los productores realizan la actividad de la ganadería sin ninguna tecnificación, lo que supone un manejo inadecuado del desarrollo de las tareas del proceso, lo que no permite aumentar la producción para mejorar sus condiciones de vida. Así mismo, se tiene que un $11 \%$ de los 
productores tiene un método semitecnificado lo que indica que cuentan con algunos medios para una mejorar producción de bovinos de ceba.

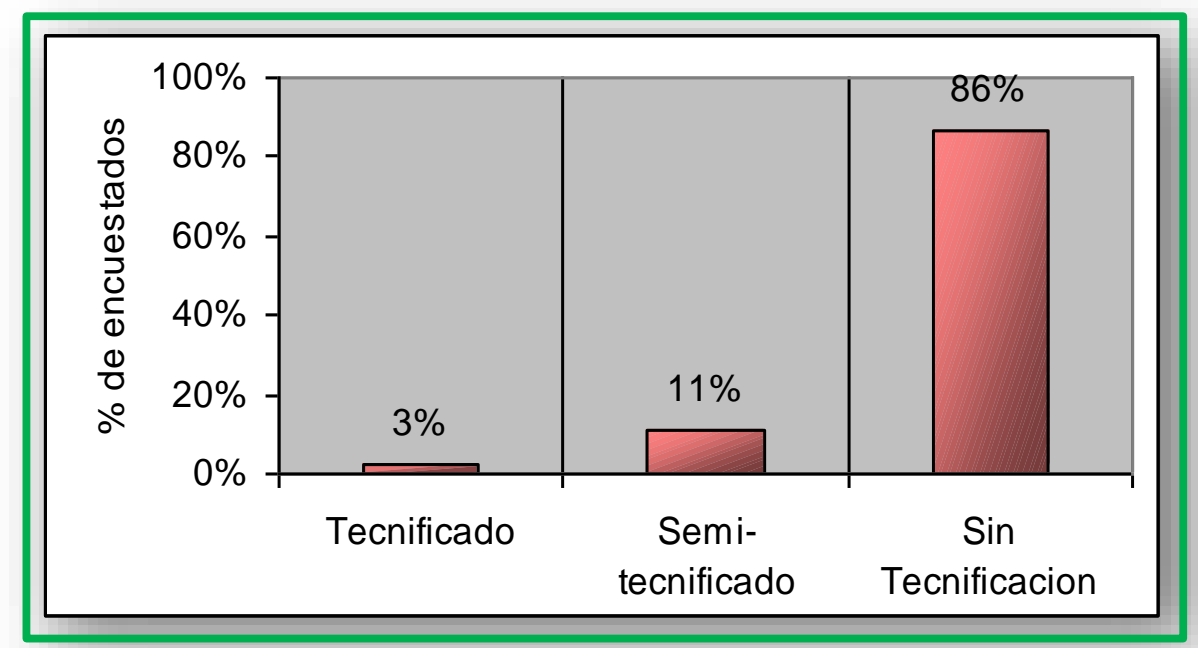

Gráfica 2. ¿El manejo de los bovinos se realiza?

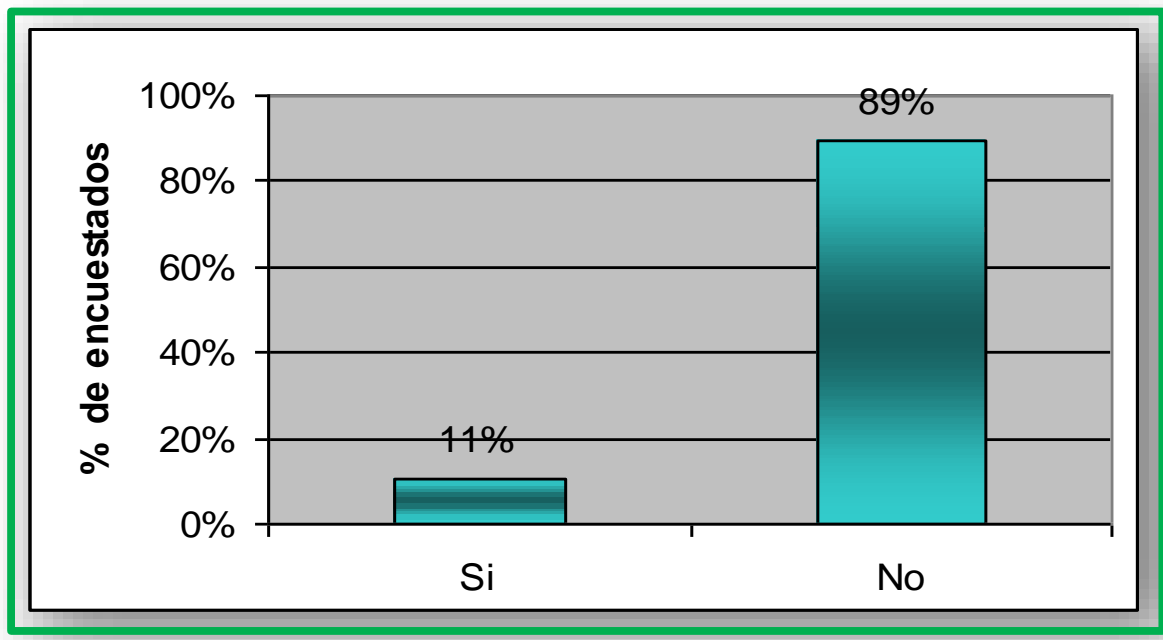

Gráfica 3. ¿Desarrolla alguna variedad de pastos?

Según los resultados de esta pregunta, un $89 \%$ de los productores de bovinos dicen que, no desarrollan ninguna variedad de pastos, lo que significa que los bovinos se están cebando con las pasturas normales producidas por el terreno y que los están rotando constantemente para poder sostener la permanencia de los pastos en las áreas en las que se divide, para ello. También se encontró que un 
$11 \%$ de los encuestados producen alguna variedad y por lo general se utiliza el Brachiaria, pero indican que es muy costoso.

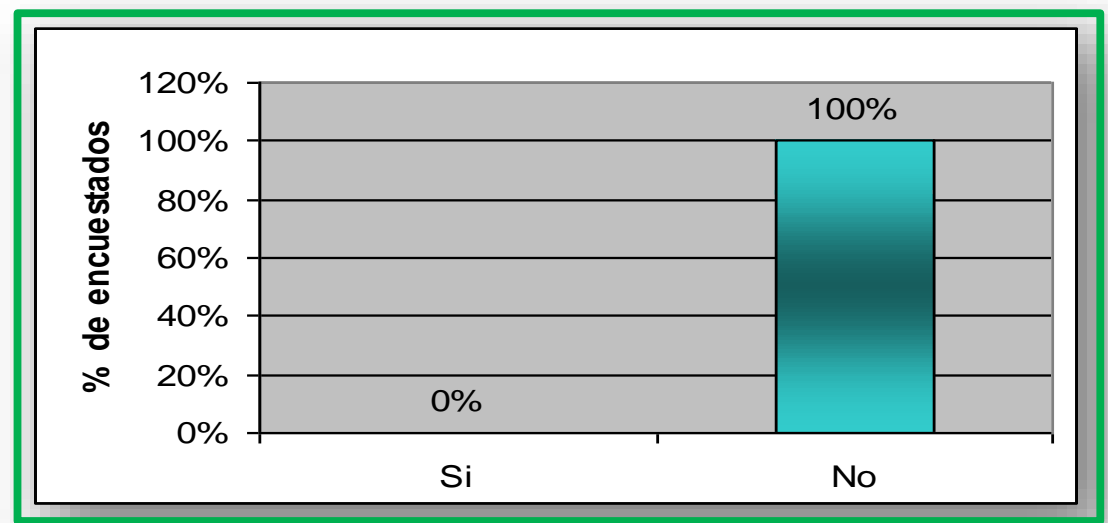

Gráfica 4. ¿Además de los pastos de pradera, les complementa la alimentación a los bovinos con otro forraie?

Los resultados a la pregunta, indican que el $100 \%$ de los productores no complementan la alimentación de los animales con otro forraje, por lo que solo emplean las pasturas que nacen de los campos propios y por eso es que la ganadería se hace extensiva, además de que los suelos no presentan las mejores condiciones para el desarrollo de los pastos que se producen en esta área geográfica.

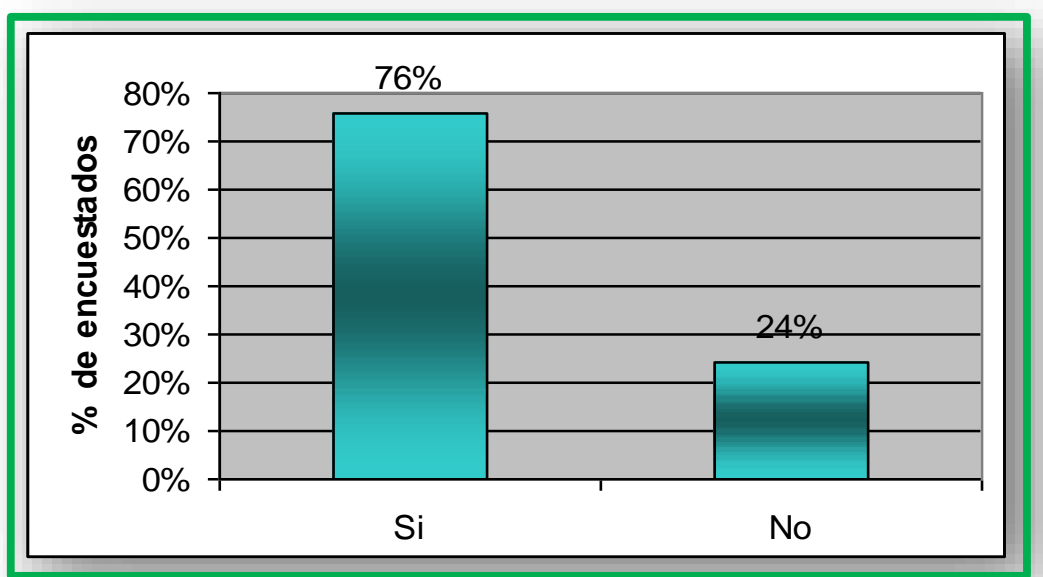

Gráfica 5. ¿La ganadería de ceba es la fuente de sus ingresos? 
El $76 \%$ de los productores depende de esta actividad como fuente de sus ingresos, por lo que se hace propicio que se estimule el desarrollo y crecimiento de esta actividad con capacitación y apoyo del Estado para mejorar la calidad de vida ya que ese es el precepto que prevalece en la Constitución.

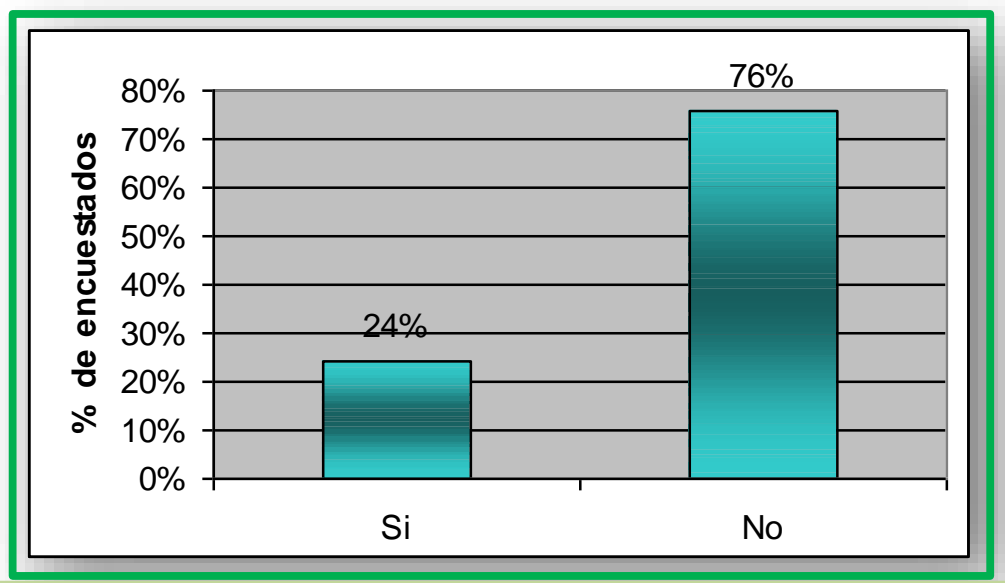

Gráfica 6. ¿Además de la ganadería de ceba, desarrolla otra actividad para generar ingresos?

El $76 \%$ de los productores de bovinos de ceba dicen que no desarrollan otra actividad para generar ingresos y que si lo hacen es para consumo familiar y que por lo tanto es indispensable avanzar en las técnicas del manejo de los animales que permitirán mejorar el nivel de vida de las personas encargadas de esta actividad.

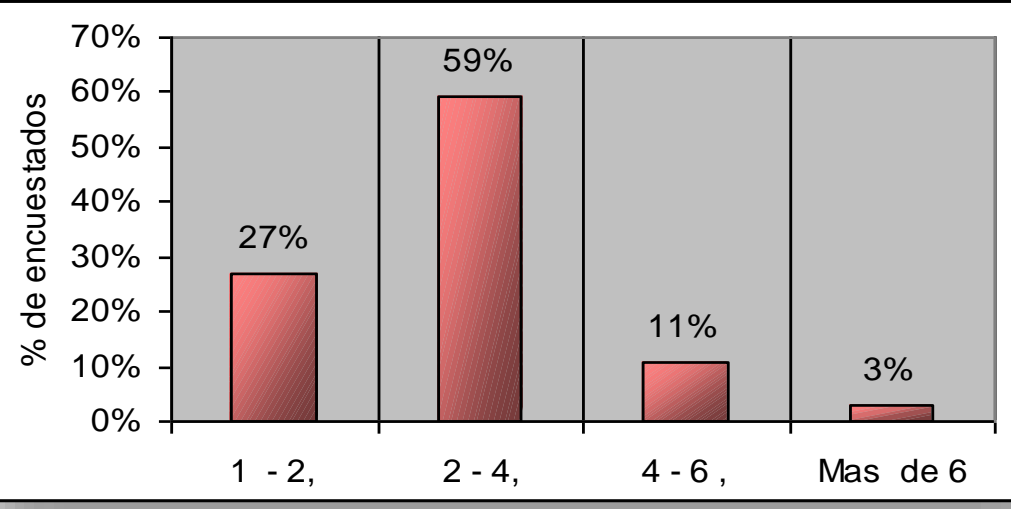

Gráfica 7. ¿Cuantos animales, ceba por hectárea de pastos? 
El 59\% de los encuestados dicen que tiene entre 2 a 4 bovinos de ceba por hectárea y que es lo que comúnmente pueden producir siempre y cuando se realice una rotación para que los terrenos y pastos se mantengan. El $27 \%$ de los productores dicen que solo tiene en promedio entre 1 a 2 bovinos por hectárea porque las pasturas no permiten tener más.

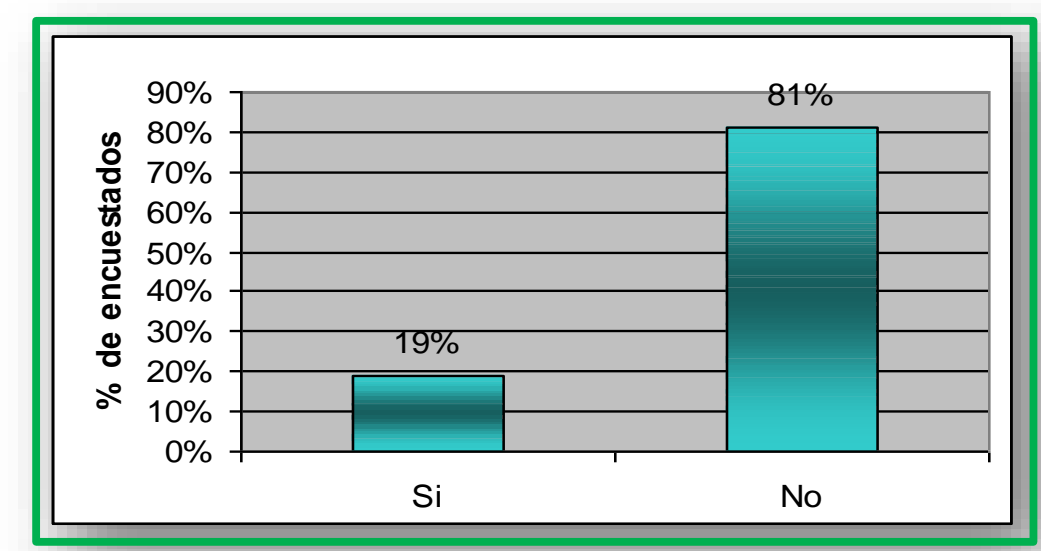

Gráfica 8. ¿Cuenta con los equipos e infraestructura adecuada para el manejo de bovinos?

El $81 \%$ de los encuestados afirman que no cuentan con los equipos e infraestructura adecuada para el manejo de bovinos y que en muchos casos hay bajas en el número de animales, lo que permite una disminución de ingresos. Así mismo, dicen que por los costos de la fabricación de establos y corrales, no se pueden desarrollar labores que se deben realizar para el cuidado de los animales lo cual indica que hay una falta de apoyo del Estado en este sector.

El $92 \%$ de los encuestados dicen que los suelos no son los adecuados para mejorar el rendimiento de los pastos ya que cada día las praderas se ven más disminuidas y erosionadas, debido a los altos costos y porque no existen conocimientos sobre el tema.

El $89 \%$ de los productores de bovinos dicen que no poseen capacitación sobre el manejo de bovinos de ceba y que todo lo que hacen es empírico y a veces con apoyo de veterinarios que contratan, pero muy de vez en cuando porque los 
costos son altos. Es importante entonces, tener en cuenta este factor, para que las personas obtengan conocimientos que le proporcionen una visión diferente de la actividad que están realizando.

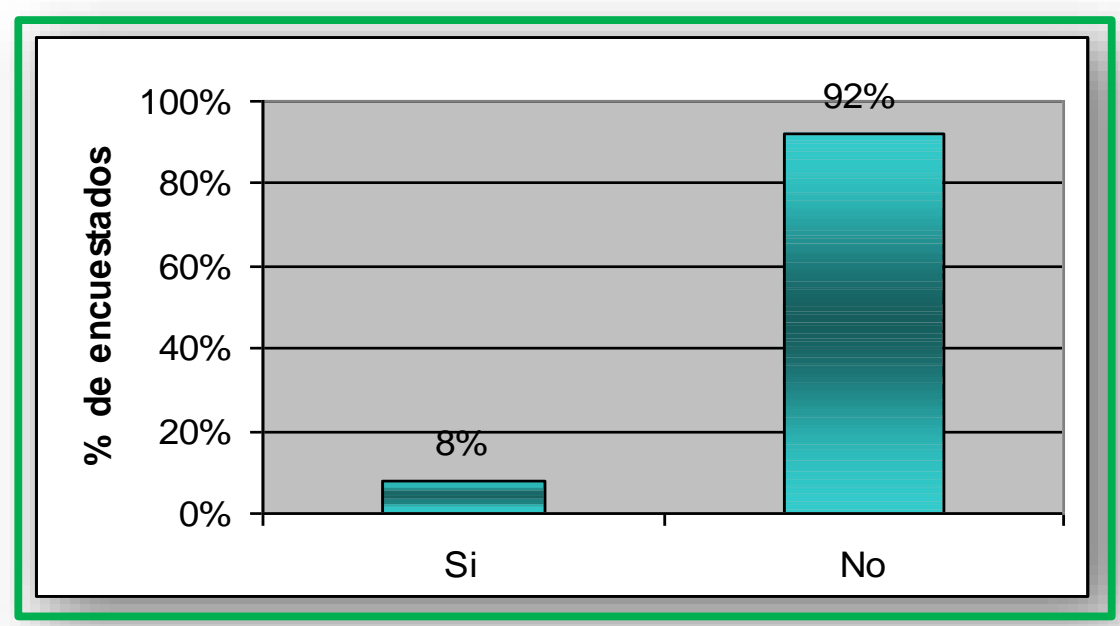

Gráfica 9. ¿Los suelos se adecuan para mejorar su rendimiento en pastos?

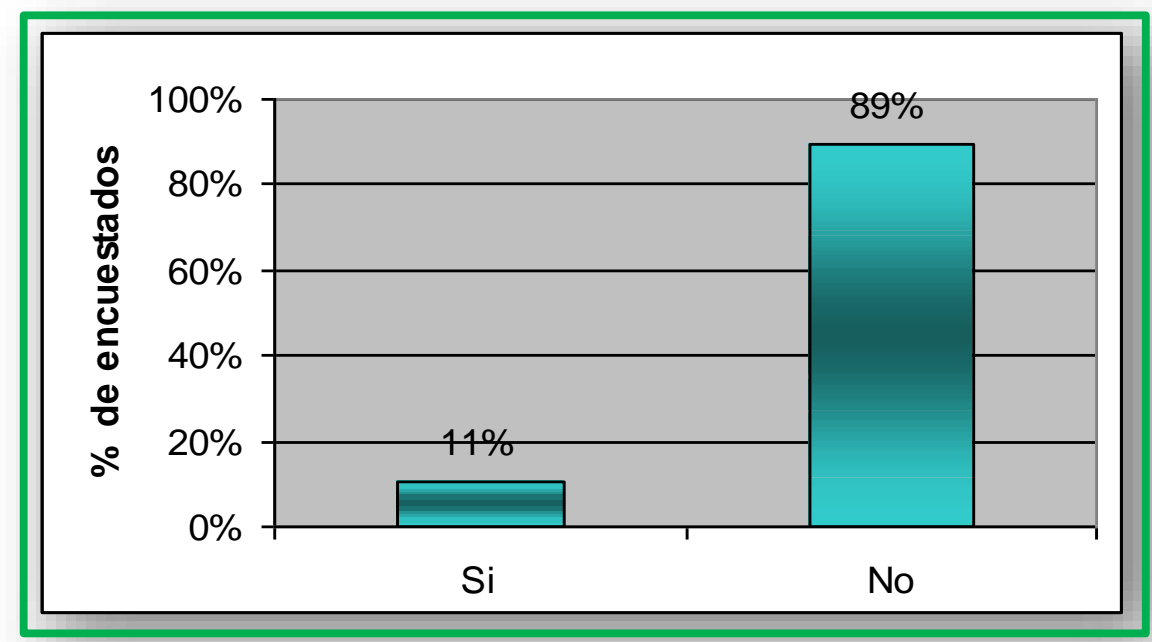

Gráfica 10. ¿Han recibido capacitación sobre el manejo de bovinos de ceba?

El 95\% de los encuestados no conoce sobre el $\mathrm{FVH}$, sin embargo el $5 \%$ que lo conocen dicen que es un alimento complementario importante para el desarrollo de los animales. Por lo cual se debe dar a conocer todos los aspectos sobre el 
tema porque sería muy interesante que ellos lo produzcan con el fin de mejorar las condiciones de ceba y poder lograr mayor rendimiento de carne en menor tiempo.

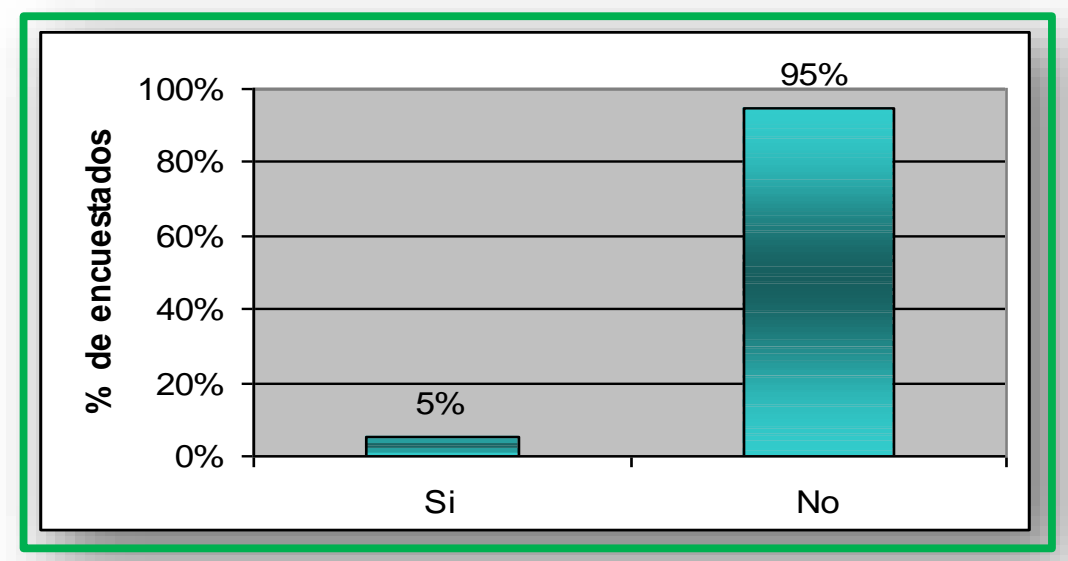

Gráfica 11. ¿Conoce sobre forraje verde hidropónico?

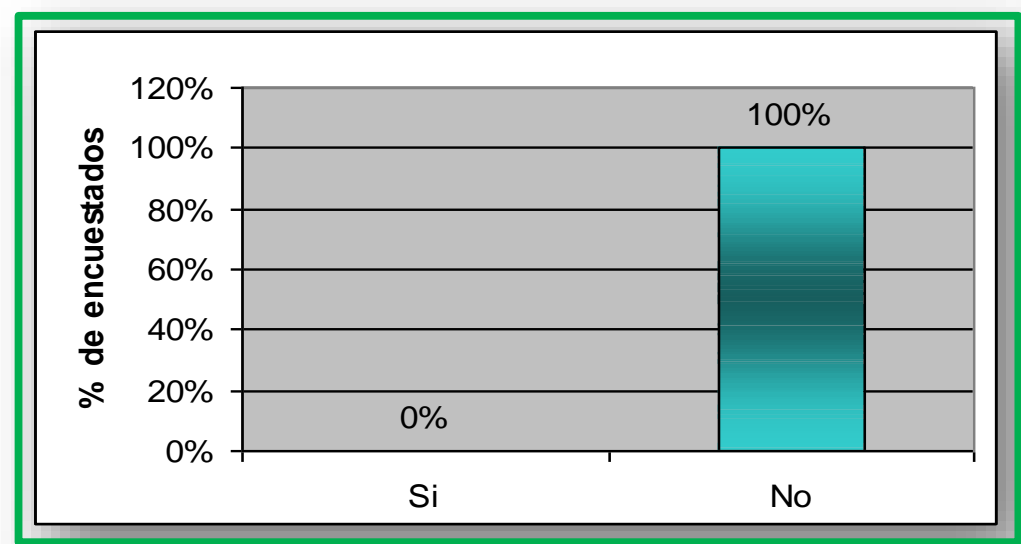

Gráfica 12. ¿El Estado los apoya en el desarrollo de su actividad ganadera?

El $100 \%$ de los encuestados dicen que no hay apoyo del Estado por medio de los tantos organismos que existen para apoyar el campo y sus actividades. $Y$ en este sentido se hace imprescindible despertar la importancia de exigir apoyo técnico y financiero para mejorar las condiciones de producción especialmente en la agricultura y ganadería que son los reglones económicos de los cuales depende la población de este sector. 
La Vereda Jiramena del Municipio de San Carlos de Guaroa en el Departamento del Meta, está ubicada en una región tropical en la cual la base de la alimentación de los bovinos la constituyen los pastos y forrajes. Por lo anterior los bovinos siempre deben tener acceso a una pastura de excelente calidad. Por eso la importancia de implementar una alimentación complementaria que permita a los productores, mejorar el desarrollo de estos animales, ya que año tras año las pasturas tropicales son afectadas por los eventos de verano o época crítica que alteran las condiciones atmosféricas, generando que los pastos se sequen y el ganado tenga poco alimento para su desarrollo productivo.

Al recolectar la información, se pudo apreciar que los ganaderos saben que esta época es crítica tanto para ellos como para los animales, por lo que se convierte en un tiempo indeseable más no inesperado, ya que se tiene pleno conocimiento de ello. Sin embargo, algunos ganaderos asumen una actitud pasiva y dejan que la sequía reduzca drásticamente sus ingresos porque afecta negativamente la producción de leche, el peso corporal y la eficiencia reproductiva en detrimento de la rentabilidad, por lo tanto, se hace indispensable el desarrollo de un sistema que proteja esos intereses.

Pero además de lo anterior, es preciso admitir que en esta región los animales con su pisoteo, en adición a la falta de un programa de mejoramiento de tierras, cada día se está perdiendo la fertilidad del suelo permitiendo que las pasturas sean mínimas en su desarrollo y los campos se formen "peladeros", en donde los animales para su alimentación diaria tienen que cubrir un área mayor y por lo tanto cada día se hace necesario tener más espacio para su producción.

En general los desbalances nutricionales ocurren en todas las épocas del año y fases productivas del animal, mientras que, en la mayoría de los agroecosistemas, la crisis nutricional se acentúa en la sequía, debido a la baja disponibilidad de forraje y a la calidad de la biomasa. Todos estos factores llevan a una baja condición corporal que trae como consecuencias el incremento del número de días abiertos, disminución del peso corporal, reducción de la tasa de preñez, incremento en el intervalo entre partos, reducción de la producción de leche, 
reducción del peso de los terneros al destete, disminución de los ingresos y la rentabilidad. La condición corporal de los animales se puede mejorar realizando una complementación alimenticia con $\mathrm{FVH}$, al igual que mejorar la calidad del forraje ofrecido en la pastura, mediante programas de rotación, fertilización, riego, etc.; complementariamente es necesario realizar control de ecto y endoparásitos, suministrar suplemento mineral adecuado, también debería suministrarse algún suplemento proteico.

En este sentido, los forrajes hidropónicos son una buena opción para la complementación alimenticia (energética-proteica) de los bovinos en la cual se aprovechan las épocas de lluvias para la siembra de pastos, maíz, millo o sorgo, que serán utilizados en el proceso hidropónico para ofrecer un forraje de buena calidad a los ganados en las épocas críticas o de verano. Los FVH aportan principalmente energía y nutrientes, lo cual es indispensable para suplir el gasto de energía de los animales. La complementación alimenticia con FVH contribuye a aumentar la digestibilidad de la fibra, permitiendo que, en las épocas críticas, el ganado no pierda peso, mientras que en período normal ayuda a la producción animal y por supuesto al aumento de peso.

El porcentaje de proteína de una muestra de FVH a los quince días es de 18.22\%. Ahora, sumado a la cantidad de proteína del pasto tradicional que según los datos mencionados (Pérez y Lascano, 1989) el pasto Brachiaria en los Llanos Orientales de Colombia, tiene 3.5\% de proteína y como consecuencia, las ganancias de peso son bajas y solo se puede llegar a mantener 1.0 unidad animal por hectárea dando un aumento de peso de $130 \mathrm{~g} / \mathrm{animal} / \mathrm{día}$ en promedio; lo que significa que el resultado sería de 807 g/animal/día complementándole la alimentación con FVH.

En resumen, se podría decir que un bovino alimentado con $\mathrm{FVH}$, gana más peso a diario y por lo tanto el periodo de ceba se disminuye considerablemente lo que permitiría obtener mayores ingresos porque se estaría rotando más rápidamente la producción. En este sentido, la producción de FVH es una de las dos megatendencias mundiales en alimentos libre de químicos y mayor producción en menor espacio, por lo que tiene un valor agregado alimenticio fundamental en el 
proceso de ceba de los animales bovinos, que además no tiene implicaciones ecológicas, pues las yerbas y plantas que servirán de alimento para el ganado pueden cultivarse sin sustancias químicas lo cual beneficiará a los animales y a los humanos que posteriormente los consuman.

\section{CONCLUSIONES}

El FVH es un sistema de alimentación complementaria creado para eliminarle al ganadero la dependencia y limitación que generan la pobreza de suelo y condiciones climatológicas adversas, tales como sequías, falta de lluvia, friaje, inundaciones, etc.

La comparación bromatológica entre los pastos tradicionales y el FVH, establece una gran diferencia en cantidad de nutrientes que permiten una buena ganancia de peso en los animales de ceba, o en mantenerlos en tiempo de verano.

El FVH posibilita que el ganadero cuente con un forraje en la cantidad deseada según la necesidad, siendo de alta calidad y a un valor sustancialmente más económico que el forraje convencional; sustituyendo así los grandes espacios de terreno que son imprescindibles para obtener forraje, creando granjas competitivas de reducidas dimensiones y altas producciones en las zonas donde el suelo y el clima son adversos.

Es factible desarrollar los invernaderos del $\mathrm{FVH}$, porque sus costos son recuperables y además permite generar utilidades propicias que les permiten suplir sus necesidades y expectativas de vida.

El sistema de FVH para este sector es un método de producción ideal para implementar en las fincas que poseen extensiones pequeñas, ya que los campos se encuentran estériles por lo que se logra una mayor utilización de las tierras y un mejor manejo de los bovinos de ceba, actividad que se desarrolla en esta área geográfica. 


\section{RECOMENDACIONES}

Realizar una asociación de ganaderos de la región con el fin de solicitar apoyo al Estado, el cual debe de proporcionar las condiciones adecuadas para el mejoramiento de vida de la población del sector, y entre ellas debe proporcionar capacitación, inversión y facilidad en obtener empréstitos que le permitan avanzar en su actividad económica. Con la misma organización, los productores podrían desarrollar un invernadero de grandes proporciones que satisfaga la necesidad de complementar la alimentación de los bovinos de ceba de todas las fincas, lo cual permitiría disminuir los costos y disponer de forraje verde en cualquier época del año, con el fin de mejorar la calidad del ganado que se maneja.

Se recomienda que en todo momento del proceso de producción de FVH, se realice un control fitosanitario eficiente con el fin de proteger la calidad del mismo y no permitir la proliferación de hongos, microorganismos que más afectan este tipo de producto.

Es importante seguir con la capacitación sobre este tema, con el objeto de optimizar el proceso y lograr los mayores resultados posibles en la producción del forraje, porque con ello se llegará a la generación de mayores utilidades y por lo tanto al incremento de la producción de bovinos de ceba.

\section{REFERENCIAS BIBLIOGRÁFICAS}

1. Acosta F. La huerta escolar autosuficiente. Libro texto universidad de los llanos. Villavicencio. p 150. 1997.

2. Acosté I. Sugerencias para enfrentar mejor la crisis. Revista del Plan Agropecuario $\mathrm{N}^{\circ}$ 89. Montevideo, Uruguay. p 14. 1999.

3. Acosté F. La extensión agrícola rural. Material de consulta. Organización de las Naciones Unidades para la agricultura y la alimentación. Roma. p 286. 1987.

4. Álvarez M., Villamizar G. Guía metodología para la formación de trabajo con grupos de pequeños productores. Programa de desarrollo tecnológico para el medio rural. Junta de acuerdo de Cartagena. Edición. Divulgación ICA Bucaramanga. p 31. 1989.

5. Arano C. Forraje verde hidropónico y otras técnicas de cultivos sin tierra. Editado por el propio autor. Providencia de Buenos. Aires, Argentina. p 78. 1998. 
6. Becth, G. System teory, the key to holimand reductionism. Bioscience. p 569579. 1974.

7. Carámbula, M; Terra, J. Las Sequías: Antes, durante y después. INIA, Treinta y tres. Montevideo, Uruguay. p 15. 2000.

8. Carámbula M. Producción y manejo de pasturas sembradas. Editorial Hemisferio Sur. Montevideo, Uruguay. p 45. 1977.

9. Carrasco G., Izquierdo J. La empresa hidropónica de mediana escala: La técnica de la solución nutritiva recirculante ("NFT"). FAO - Univ. De Talca. Santiago, Chile. p 78. 1996.

10. Church D. C. Fisiología digestiva y nutrición de los rumiantes. Editorial Acribia. Zaragoza, España. p 45. 1974.

11. Chang M., Rodríguez D. A., Hoyos R. Producción de forraje verde hidropónico. $S$ Manual práctico de hidroponía. Centro de Investigación de Hidroponía y Nutrición Mineral. UNALM. Lima, Perú. p 100. 2001.

12. Dosal A. J. M. Efecto de la dosis de siembra, época de cosecha y fertilización sobre la calidad y cantidad de forraje de avena producido bajo condiciones de hidroponía. p 99. 1987.

13. FAO. Forraje verde hidropónico, Manual técnico. Oficina regional para América Latina y El Caribe. Santiago, Chile. p 79. 2002.

14. Fox R. Fábrica de forraje. Boletín Informativo de la Red Hidroponía № 8. Lima, Perú. p 12. 2000.

15. Gastal E. Sistemas de producto. En: Sistemas de producción agrícola, mesa redonda del consejo técnico consultivo. XXII Reunión. Santo Domingo. República Dominicana. IICA. p 45. 1977.

16. Harris W. Pasture as an ecosystem. Edit. R.H.M. Oxford, University. p 34. 1990.

17. Hidalgo M. L. R. Producción de forraje en condiciones de hidroponía. Evaluaciones preliminares en avena y triticale. Facultad de Ciencias Agropecuarias y Forestales de la Universidad de Concepción, sede Chillan. Chile. p 102. 1985.

18. INDAP. Consultoría de Forrajes Hidropónicos. Servicio de Información para la Agricultura. Chile. p 89. 2003.

19. Méndez A. C. E. Metodología, guía para elaborar diseños de investigación en ciencias económicas, contables y administrativas. Bogotá. Editorial Mc Graw Hill. p.123. 1998.

20. Pérez R. A., Lascano C. E. El pasto Brachiaria en los Llanos Orientales de Colombia. Boletín técnico № 181. Divulgación ICA. Villavicencio., p 10-15. 1989.

21. Sánchez, A. Una experiencia de forraje verde hidropónico en el Uruguay. En Boletín Informativo Red Hidroponía No 7. CIHNM. UNALM. Lima, Perú. p 36. 2000.

22. Saravia A. Un enfoque de sistemas para el desarrollo agrícola. San José. Costa Rica. IICA., p 41-50. 1985.

23. Tarrillo, H. Producción de Forraje Verde Hidropónico en Arequipa, Perú. En: Boletín Informativo de Red Hidroponía. CIHNM, UNALM. Lima, p 20. 2002. 